\title{
Absolute bunch length measurements by incoherent radiation fluctuation analysis
}

\author{
F. Sannibale, ${ }^{1, *}$ G. V. Stupakov, ${ }^{2}$ M. S. Zolotorev, ${ }^{1}$ D. Filippetto, ${ }^{3}$ and L. Jägerhofer ${ }^{4}$ \\ ${ }^{1}$ Lawrence Berkeley National Laboratory, One Cyclotron Road, Berkeley, California 94720, USA \\ ${ }^{2}$ SLAC National Accelerator Laboratory, Menlo Park, California 94025, USA \\ ${ }^{3}$ INFN-LNF, Frascati, Rome, Italy \\ ${ }^{4}$ University of Technology Vienna, Vienna, Austria \\ (Received 29 September 2008; published 4 March 2009)
}

\begin{abstract}
By analyzing the pulse to pulse intensity fluctuations of the radiation emitted by a charge particle in the incoherent part of the spectrum, it is possible to extract information about the spatial distribution of the beam. At the Advanced Light Source of the Lawrence Berkeley National Laboratory, we have developed and successfully tested a simple scheme based on this principle that allows for the absolute measurement of the rms bunch length. A description of the method and the experimental results are presented.
\end{abstract}

DOI: 10.1103/PhysRevSTAB.12.032801

\section{INTRODUCTION}

Charged particle beams can radiate in many ways, by synchrotron radiation, Cerenkov radiation, transition radiation, etc. In all radiating processes the presence of incoherent radiation is due to the fact that the particles are randomly distributed along the beam.

For example, in the case of an ideal coasting beam composed of a large number of particles equally separated by a longitudinal distance $d$ and moving along a circular trajectory, there is no synchrotron radiation emission apart at those wavelengths where $\lambda=n d$, with $n$ being an integer. For those wavelengths the radiation from different particles is in phase and the emission is fully coherent. For all other wavelengths, the interference between the radiation emitted by the evenly distributed electrons will produce a vanishing net radiation field.

In a more realistic coasting beam, the same particles are instead randomly distributed along the orbit causing a small modulation of the beam distribution. The effect of such a random modulation is that the interference is not fully destructive and a net nonzero radiation field shows up. Let us now assume that at some fixed azimuthal position along the trajectory and synchronously with the revolution period of the particles, we measure the radiation emitted by the beam during a fixed time window. If the position of the particles along the beam does not change from turn to turn, then the intensity and the spectrum of the collected radiation will be constant turn to turn. If instead, as in the real case, the mutual position of particles in the bunch changes, due for instance to longitudinal dispersion or to path length dependence on transverse position, then the modulation changes as well, and the energy radiated in a single pass and its spectrum fluctuate turn by turn. In this last case, by measuring the radiation over multiple passages, we would observe that for a sufficiently large num-

*FSannibale@LBL.gov
PACS numbers: 41.75.Ht, 41.60.- m, 05.40.- a, 06.30.- $\mathrm{k}$

ber of samples, the measured average spectrum converges to the characteristic incoherent spectrum of the radiation process under observation (synchrotron radiation from a dipole in our example).

The passage from the coasting to the bunched beam case introduces a strong coherent emission at those wavelengths comparable to or longer than the bunch length, but does not modify the short wavelength part of the spectrum.

It has been shown [1] for the case of a bunched beam that, by measuring the pulse to pulse spectrum fluctuation of the radiation emitted by the beam in a region of the spectrum where no coherent emission is present, it is possible to perform absolute measurements of the bunch length. Schemes exploiting such a technique and using high resolution spectrometers or interferometers have been proposed and already proved experimentally [2-5].

At the Lawrence Berkeley National Laboratory (LBNL), we have developed a remarkably simpler new version that does not require complex and expensive instrumentation and that allows for accurate absolute measurements of the rms bunch length. In such a scheme the radiation intensity emitted during a single beam passage is measured within a fixed bandwidth $\Delta \omega$ in the incoherent part of the spectrum. The bunch length information is then extracted by the analysis of the turn by turn intensity fluctuation. The technique can use any kind of radiating process as long as $\Delta \omega$ is much smaller than the spectral width of the radiating process, and the electron beam angular spread is much smaller than the radiation angular distribution width at the wavelength of interest. It is worth remarking that the two conditions above can be easily matched in most of the cases. The method is applicable to both circular and linear accelerators including cases where the very short length of the bunches makes difficult the use of other techniques. The measurement requires multiple intensity acquisitions, and if the bunch length changes during the measure, the fluctuation analysis will give the average bunch length over the data taking period. Shot to shot 
charge variations, typical of linac accelerators, for example, can be properly handled by a simple modification of the system as explained in the last section of this paper.

A bunch length monitor based on such a scheme and using visible synchrotron radiation from a dipole magnet was developed and successfully tested at the Advanced Light Source (ALS), the LBNL third generation light source [6]. In this paper, the measurement theory is presented and the results are then used for explaining the bunch length measurements performed on the ALS beam.

\section{THEORY}

We assume that each particle in a bunched beam radiates an electromagnetic pulse in the direction characterized by the angles $\theta_{x}$ and $\theta_{y}$, with the electric field given by a function $e(t, \boldsymbol{\theta})$, where $t$ is time and $\boldsymbol{\theta}=\left(\theta_{x}, \theta_{y}\right)$ the angle with respect to the direction of motion of the beam. The exact expression for this function depends on the radiation process involved and is not essential for our calculations. We also assume that we are interested in radiation at small angles with respect to the direction of motion of the beam, $\left|\theta_{x}\right|,\left|\theta_{y}\right| \ll 1$. Note that using the same angular distribution for the radiation field in the function $e(t, \boldsymbol{\theta})$ we neglect the angular spread of the electrons in the beam [7]. This is justified if the angular spread of electrons is much smaller than the characteristic angles of the radiation at the wavelength of interest, which is generally satisfied in the experiment type described in this paper.

The total electric field of the radiation of the beam is a sum of the fields radiated by each electron. If the longitudinal position of $k$ th particle within the bunch is marked by the time variable $t_{k}$, and the transverse coordinates are $x_{k}$ and $y_{k}$, the total radiated field $E(t, \boldsymbol{\theta})$ propagating at angles $\theta_{x}$ and $\theta_{y}$ is

$$
E(t, \boldsymbol{\theta})=\sum_{k=1}^{N} e\left(t-t_{k}-\frac{1}{c} \boldsymbol{r}_{k} \cdot \boldsymbol{\theta}, \boldsymbol{\theta}\right)
$$

where $N$ is the total number of particles in the bunch. In this equation we introduced the two-dimensional vectors $\boldsymbol{r}_{k}=\left(x_{k}, y_{k}\right)$ defining the transverse position of the $k$ th particle. The first argument in the function $e$ takes into account that the path length for the radiation field, and hence the delay time, depends on the position $\boldsymbol{r}_{k}$ and the arrival time $t_{k}$ of particle $k$, as well as on the observation angles $\theta_{x}$ and $\theta_{y}$. We assume that $t_{k}$ are random numbers, with the probability to find $t_{k}$ between $t$ and $t+d t$ equal to $f_{t}(t) d t$, where $f_{t}(t)$ is the bunch distribution function [normalized so that $\left.\int_{-\infty}^{\infty} f_{t}(t) d t=1\right]$. Analogously, the transverse position of a particle is defined by the distribution functions $F(\boldsymbol{r})$ [where $\boldsymbol{r}=(x, y)$ ] normalized in such a way that $\int F(\boldsymbol{r}) d x d y=1$. In the context of a bunched beam with a given beam current $I(t)$, the longitudinal distribution function can be understood as a normalized function $I$ :

$$
f_{t}(t)=I(t)\left(\int_{-\infty}^{\infty} I(t) d t\right)^{-1}
$$

For a Gaussian distribution, $f_{t}(t)=\left(2 \pi \sigma_{t}^{2}\right)^{-1 / 2} e^{-t^{2} / 2 \sigma_{t}^{2}}$, where $\sigma_{t}$ is the rms bunch length in units of time. Similarly, for transverse Gaussian distributions $\sigma_{x}$ and $\sigma_{y}$ define the rms transverse sizes of the bunch. We also assume that positions of different particles in the bunch, $k$ and $i$ for $k \neq i$, are uncorrelated: $\left\langle t_{k} t_{i}\right\rangle=\left\langle t_{k}\right\rangle\left\langle t_{i}\right\rangle,\left\langle x_{k} x_{i}\right\rangle=$ $\left\langle x_{k}\right\rangle\left\langle x_{i}\right\rangle,\left\langle y_{k} y_{i}\right\rangle=\left\langle y_{k}\right\rangle\left\langle y_{i}\right\rangle$, with the angular brackets denoting the averaging.

The spectral properties of the radiation are related to the Fourier transform $\hat{E}(\omega, \boldsymbol{\theta})$ of the field:

$$
\hat{E}(\omega, \boldsymbol{\theta})=\int_{-\infty}^{\infty} E(t, \boldsymbol{\theta}) e^{i \omega t} d t=\hat{e}(\omega, \boldsymbol{\theta}) \sum_{k=1}^{N} e^{i \omega t_{k}+i \omega r_{k} \cdot \boldsymbol{\theta} / c},
$$

where $\hat{e}(\omega, \boldsymbol{\theta})=\int_{-\infty}^{\infty} e(t, \boldsymbol{\theta}) e^{i \omega t} d t$.

In the experiment, the spectrum of the radiation $P(\omega, \boldsymbol{\theta})$, which is proportional to $|\hat{E}(\omega, \boldsymbol{\theta})|^{2}$ is the point of interest. A limiting aperture of the diagnostic system introduces an additional factor, which we denote by $S(\boldsymbol{\theta})$, into the measured intensity. Taking into account this aperture function and a bandpass filter with an intensity transmission function $R(\omega)$, the spectral intensity reaching the detector can be written as

$$
P(\omega, \boldsymbol{\theta})=S(\boldsymbol{\theta}) R(\omega)|\hat{E}(\omega, \boldsymbol{\theta})|^{2} .
$$

Using the notation

$$
T(\omega, \boldsymbol{\theta})=S(\boldsymbol{\theta}) R(\omega)|\hat{e}(\omega, \boldsymbol{\theta})|^{2},
$$

we find from Eq. (3)

$$
P(\omega, \boldsymbol{\theta})=T(\omega, \boldsymbol{\theta}) \sum_{k, l=1}^{N} e^{i \omega\left[t_{k}-t_{l}+\left(\boldsymbol{r}_{k}-\boldsymbol{r}_{l}\right) \cdot \boldsymbol{\theta} / c\right]}
$$

To find the average spectral intensity, we integrate Eq. (6) with the distribution functions

$$
\begin{aligned}
\langle P(\omega, \boldsymbol{\theta})\rangle= & T(\omega, \boldsymbol{\theta}) \sum_{k, l=1}^{N} \int_{-\infty}^{\infty} \int_{-\infty}^{\infty} d t_{k} d t_{l} f_{t}\left(t_{k}\right) f_{t}\left(t_{l}\right) e^{i \omega\left(t_{k}-t_{l}\right)} \\
& \times \iint d^{2} r_{k} d^{2} r_{l} F\left(\boldsymbol{r}_{k}\right) F\left(\boldsymbol{r}_{l}\right) e^{i \omega\left(\boldsymbol{r}_{k}-\boldsymbol{r}_{l}\right) \cdot \boldsymbol{\theta} / c} \\
= & N T(\omega, \boldsymbol{\theta})\left(1+N\left|\hat{f}_{t}(\omega)\right|^{2}|\hat{F}(\boldsymbol{\kappa} \boldsymbol{\theta})|^{2}\right)
\end{aligned}
$$

where $\quad \kappa=\omega / c, \quad \hat{f}_{t}(\omega)=\int_{-\infty}^{\infty} f_{t}(t) e^{i \omega t} d t, \quad \hat{F}(\boldsymbol{a})=$ $\int_{-\infty}^{\infty} F(\boldsymbol{r}) e^{i \boldsymbol{a} \cdot \boldsymbol{r}} d x d y$ are the Fourier transforms of the distribution functions [for the Gaussian distribution mentioned above, $\left.\hat{f}_{t}(\omega)=e^{-\omega^{2} / 2 \sigma_{t}^{2}}\right]$, and we set $N-1 \approx N$. The first term in Eq. (7) is incoherent radiation proportional to the number of particles in the bunch. The second term is the coherent radiation that scales quadratically with $N$. The coherent radiation term carries information about the distribution function of the beam but only at relatively low 
frequencies of the order of $\omega \lesssim \sigma_{t}^{-1}$, where $\hat{f}_{t}(\omega)$ is not zero. At high frequencies, where $N\left|\hat{f}_{t}(\omega)\right|^{2} \ll 1$, the coherent radiation is negligible in comparison with the incoherent one. In this case Eq. (7) becomes

$$
\langle P(\omega, \boldsymbol{\theta})\rangle=N T(\omega, \boldsymbol{\theta}) .
$$

However, the original, unaveraged expression for the spectral power, Eq. (6), shows that the properties of the radiation even at high frequencies carry information about the longitudinal distribution function. Indeed, each term $e^{i \omega\left(t_{k}-t_{l}\right)}$, considered separately, oscillates as a function of frequency, with the period $\Delta \omega=2 \pi /\left(t_{k}-t_{l}\right) \sim 2 \pi / \sigma_{t}$. Because the distribution of particles in the bunch randomly changes from bunch to bunch, in the case of a linear accelerator, or from turn to turn in the case of a circular accelerator, the sum in Eq. (6) fluctuates randomly as well, and statistical properties of these fluctuations depend on the distribution function of the bunch.

To obtain a quantitative characteristic of the fluctuation, we first calculate, as an intermediate step, the average value of the product $P(\omega, \boldsymbol{\theta}) P\left(\omega^{\prime}, \boldsymbol{\theta}^{\prime}\right)$ :

$$
\begin{aligned}
\left\langle P(\omega, \boldsymbol{\theta}) P\left(\omega^{\prime}, \boldsymbol{\theta}^{\prime}\right)\right\rangle= & T(\omega, \boldsymbol{\theta}) T\left(\omega^{\prime}, \boldsymbol{\theta}^{\prime}\right) \\
& \times \sum_{k, l, m, n=1}^{N}\left\langle e^{i \omega\left(t_{k}-t_{l}\right)+i \omega^{\prime}\left(t_{m}-t_{n}\right)}\right\rangle \\
& \times \sum_{k, l, m, n=1}^{N}\left\langle e^{i \kappa \boldsymbol{\theta} \cdot\left(\boldsymbol{r}_{k}-\boldsymbol{r}_{l}\right)+i \kappa^{\prime} \boldsymbol{\theta}^{\prime}\left(\boldsymbol{r}_{m}-\boldsymbol{r}_{n}\right)}\right\rangle .
\end{aligned}
$$

Assuming that $N|f(\omega)|^{2}, N\left|f\left(\omega^{\prime}\right)\right|^{2} \ll 1$ (which means that we can neglect the coherent radiation), it is straightforward to find

$$
\begin{aligned}
& \left\langle P(\omega, \boldsymbol{\theta}) P\left(\omega^{\prime}, \boldsymbol{\theta}^{\prime}\right)\right\rangle \\
& =N^{2} T(\omega, \boldsymbol{\theta}) T\left(\omega^{\prime}, \boldsymbol{\theta}^{\prime}\right) \\
& \quad \times\left[1+\left|\hat{f}_{t}\left(\omega-\omega^{\prime}\right)\right|^{2}\left|\hat{F}\left(\kappa \boldsymbol{\theta}-\kappa^{\prime} \boldsymbol{\theta}^{\prime}\right)\right|^{2}\right],
\end{aligned}
$$

where the contribution to the final result comes from terms with $k=l, m=n, k \neq m$ and $k=n, l=m, k \neq l$.

Equation (10) will be later used for calculation of the radiated energy $\mathcal{E}$, which is given by

$$
\mathcal{E}=\int_{-\infty}^{\infty} P(\omega, \boldsymbol{\theta}) d \omega d \theta_{x} d \theta_{y}
$$

with the average value:

$$
\langle\mathcal{E}\rangle=\int_{-\infty}^{\infty}\langle P(\omega, \boldsymbol{\theta})\rangle d \omega d \theta_{x} d \theta_{y} .
$$

To calculate the fluctuation of the radiated energy $\Delta \mathcal{E}=$ $\mathcal{E}-\langle\mathcal{E}\rangle$ we will compute the quantity

$$
\begin{aligned}
\delta^{2} & =\frac{\left\langle\Delta \mathcal{E}^{2}\right\rangle}{\langle\mathcal{E}\rangle^{2}} \\
& =\langle\mathcal{E}\rangle^{-2} \int_{-\infty}^{\infty}\left\langle[P-\langle P\rangle]\left[P^{\prime}-\left\langle P^{\prime}\right\rangle\right]\right\rangle d \omega d \theta_{x} d \theta_{y} d \omega^{\prime} d \theta_{x}^{\prime} d \theta_{y}^{\prime} \\
& =\langle\mathcal{E}\rangle^{-2} \int_{-\infty}^{\infty}\left[\left\langle P P^{\prime}\right\rangle-\langle P\rangle\left\langle P^{\prime}\right\rangle\right] d \omega d \theta_{x} d \theta_{y} d \omega^{\prime} d \theta_{x}^{\prime} d \theta_{y}^{\prime},
\end{aligned}
$$

where we used the notation $P=P(\omega, \boldsymbol{\theta})$ and $P^{\prime}=$ $P\left(\omega^{\prime}, \boldsymbol{\theta}^{\prime}\right)$. We now use Eqs. (8) and (10) for the average power $\langle P\rangle$ and the averaged product $\left\langle P P^{\prime}\right\rangle$ and obtain

$$
\begin{aligned}
\delta^{2}= & \left(\int_{-\infty}^{\infty} T d \omega d \theta_{x} d \theta_{y}\right)^{-2} \int_{-\infty}^{\infty} T T^{\prime}\left|\hat{f}_{t}\left(\omega-\omega^{\prime}\right)\right|^{2} \\
& \times \mid \hat{F}\left(\kappa \boldsymbol{\theta}-\left.\kappa^{\prime} \boldsymbol{\theta}^{\prime}\right|^{2} d \omega d \theta_{x} d \theta_{y} d \omega^{\prime} d \theta_{x}^{\prime} d \theta_{y}^{\prime} .\right.
\end{aligned}
$$

The integrals in Eq. (14) can be easily calculated if we assume for the beam Gaussian distribution functions with the rms bunch length $\sigma_{t}$ and the rms transverse sizes $\sigma_{x}$ and $\sigma_{y}$,

$$
\hat{f}_{t}(\omega)=e^{-\omega^{2} / 2 \sigma_{t}^{2}}, \quad \hat{F}(\kappa \boldsymbol{\theta})=e^{-\kappa^{2} \theta_{x}^{2} \sigma_{x}^{2} / 2-\kappa^{2} \theta_{y}^{2} \sigma_{y}^{2} / 2,}
$$

and also assume Gaussian profiles for the function $T$ :

$$
T(\omega, \boldsymbol{\theta})=T_{0} e^{-\left(\omega-\omega_{0}\right)^{2} / 2 \sigma_{\omega}^{2}-\theta_{x}^{2} / 2 \sigma_{\theta_{x}}^{2}-\theta_{y}^{2} / 2 \sigma_{\theta_{y}}^{2}},
$$

where $\omega_{0}$ and $\sigma_{\omega}$ are, respectively, the central frequency of the spectrum and its rms width, and $\sigma_{\theta_{x}}$ and $\sigma_{\theta_{y}}$ are the rms angular spreads of the radiation reaching the detector, and where we have assumed that the filter bandwidth $\sigma_{\omega}$ is much smaller than the spectral width of the radiation.

We will also assume that the width of the spectral window is small so that the following two conditions are satisfied: $\sigma_{\omega} \ll c / \sigma_{x} \sigma_{\theta_{x}}$ and $\sigma_{\omega} \ll c / \sigma_{y} \sigma_{\theta_{y}}$. In this case, we can approximately replace $\kappa \boldsymbol{\theta}-\kappa^{\prime} \boldsymbol{\theta}^{\prime} \rightarrow \kappa_{0}(\boldsymbol{\theta}-$ $\left.\boldsymbol{\theta}^{\prime}\right)$ in Eq. (14), with $\kappa_{0}=\omega_{0} / c$. Then the integration over angles decouples from the integration over the frequency and the result is

$$
\delta^{2}=\frac{1}{\sqrt{1+4 \sigma_{\omega}^{2} \sigma_{t}^{2}} \sqrt{1+4 \kappa_{0}^{2} \sigma_{\theta_{x}}^{2} \sigma_{x}^{2}} \sqrt{1+4 \kappa_{0}^{2} \sigma_{\theta_{y}}^{2} \sigma_{y}^{2}}} .
$$

A more general case of arbitrary angular and spectral distributions as well as arbitrary beam distribution functions is considered in the Appendix.

\section{PHYSICAL INTERPRETATION AND POSSIBLE APPLICATION}

Expression (17) shows the potential of fluctuation analysis for measuring the absolute length of a bunch. For the moment, let us assume that the transverse size of the beam is small enough so that Eq. (17) can be written as 


$$
\delta^{2} \simeq \frac{1}{\sqrt{1+4 \sigma_{\omega}^{2} \sigma_{t}^{2}}} .
$$

If we use a bandpass filter to define the frequency acceptance of the system, then $\sigma_{\omega}$ is known, and by measuring $\delta$, it is possible to derive the absolute value of the rms bunch length. For $\sigma_{t} \gg 1 /\left(2 \sigma_{\omega}\right)$, we have that Eq. (18) becomes $\delta^{2} \simeq 1 /\left(2 \sigma_{\omega} \sigma_{t}\right)$, and using the fact that the longitudinal coherence length of an electromagnetic mode with frequency content $\sigma_{\omega}$ is $\sigma_{t c}=1 /\left(2 \sigma_{\omega}\right)$ [8], we can write

$$
\delta^{2} \simeq \frac{\sigma_{t c}}{\sigma_{t}}=\frac{1}{M},
$$

where $M$ is the number of modes contained in the bunch. Equation (19) leads to the physical interpretation that the intensity fluctuation is due to $M$ independent longitudinal modes radiating randomly within the bunch. Indeed, the radiation from a single mode is a stochastic Poisson process whose intensity shows $100 \%$ fluctuation $[9,10]$. When $M$ independent modes radiate in a combined way, the resulting fluctuation scales as described by Eq. (19).

Equation (19) also shows that, for a fixed $\sigma_{\omega}$, a shorter bunch yields larger fluctuation $\delta^{2}$. In other words, shorter bunches are easier to measure and there is no limit on how short the bunch can be as long as no coherent radiation component is present within the frequency bandwidth used in the measurement.

For the more general case of a finite transverse beam size, we can define the transverse coherence lengths as $\sigma_{x c}=1 /\left(2 k_{0} \sigma_{\theta_{x}}\right)$ and $\sigma_{y c}=1 /\left(2 k_{0} \sigma_{\theta_{y}}\right)$ analogously to the longitudinal case. Using these quantities, Eq. (17) assumes the shape

$$
\begin{aligned}
\delta^{2} & =\frac{1}{\sqrt{1+\sigma_{t}^{2} / \sigma_{t c}^{2}} \sqrt{1+\sigma_{x}^{2} / \sigma_{x c}^{2}} \sqrt{1+\sigma_{y}^{2} / \sigma_{y c}^{2}}} \\
& =\frac{1}{\sqrt{1+M^{2}} \sqrt{1+M_{x}^{2}} \sqrt{1+M_{y}^{2}}},
\end{aligned}
$$

where we have indicated with $M_{x}$ and $M_{y}$ the number of transverse modes in the horizontal and vertical planes, respectively. Equation (20) shows that the effect of finite transverse planes is to add additional modes radiating independently. The transverse coherence sizes are defined by the wavelength of the photons and by the properties of the radiation process used in the measurement and must include diffraction effects due to limiting apertures. For simple cases analytical expressions for $\sigma_{x c}$ and $\sigma_{y c}$ can be derived and for more complex cases radiation wavefront calculation codes as SRW [11], for example, can be used.

The elegant and simple shape of Eq. (20) has been obtained assuming Gaussian distributions for the beam sizes and for the radiation distribution. For non-Gaussian distributions Eq. (14) needs to be integrated numerically.
However, it can be shown that the longitudinal term in Eq. (20) can still be used with a few percent accuracy for most of the beam distributions, as long as they are represented by their rms length and do not include microstructures with characteristic length much smaller than the bunch length. Additionally, in the typical beam line case where the photon fan is defined by an aperture, the angular photon distribution is mainly shaped by the diffraction through the aperture. In this situation, the transverse mode coherent profiles are described with very good approximation by the $[\sin (\xi) / \xi]^{2}$ function typical of a plane wave diffracting through a finite aperture. Calculations show that, in this case, it is still possible to use the two transverse terms in Eq. (20) if one fits the central peak of the $[\sin (\xi) / \xi]^{2}$ function with a Gaussian, and uses for the angular distribution width the rms width of the fit divided by $\sqrt{2}$. In other words, Eq. (20) can be used in a large number of non-Gaussian cases of practical interest still maintaining a few percent accuracy.

\section{EXPERIMENTAL CONSIDERATIONS AND THE ADVANCED LIGHT SOURCE MEASUREMENTS}

Figure 1 shows the experimental setup for the measurements performed at the IR-visible branch line of beam line BL 7.2 of the ALS. Such a beam line collects the synchrotron radiation from a dipole magnet and has a total angular acceptance of 5.5 and $2.8 \mathrm{mrad}$ for the horizontal and vertical planes, respectively (represented in Fig. 1 by the limiting aperture). As required by the measurement theory, such values are much larger than the rms angular spread of the electron beam at the BL 7.2 source point. Indeed, accounting for the ALS emittance and optical functions, such a spread was always smaller than $\approx 50 \mu \mathrm{rad}$ for all measurement conditions. The radiation spectrum at this branch line is limited by design within a frequency interval

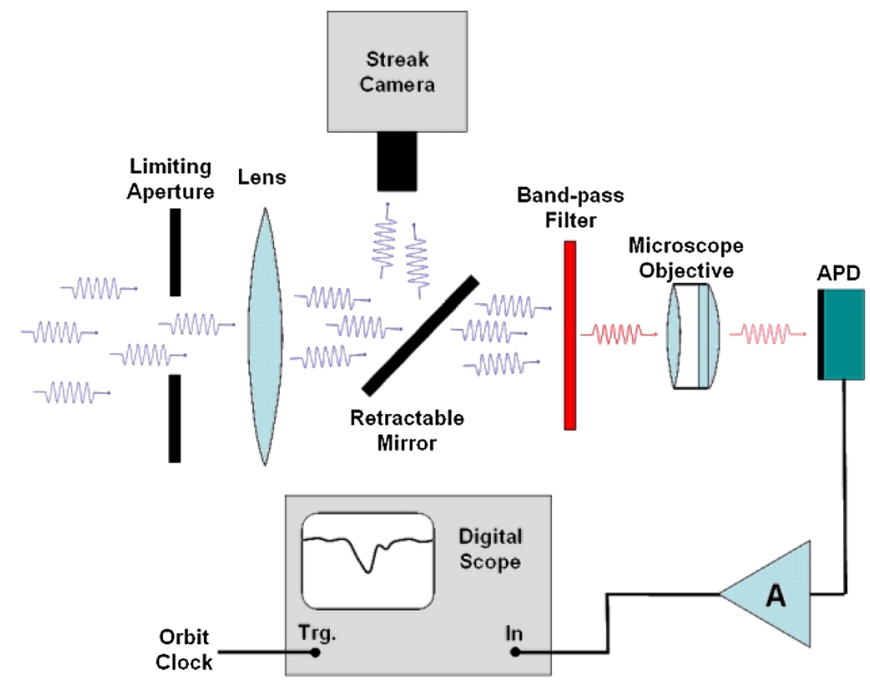

FIG. 1. (Color) Schematic diagram of the setup used for the experiment at the ALS. 
ranging from the far infrared up to the visible. In our application we used visible light because it is in the incoherent part of the spectrum, as required, and also because of the large variety and relatively inexpensive optical components and detectors readily available for such a frequency range. A $1 \mathrm{~m}$ focal length lens was used to focus, through a flat mirror, the beam on the photocathode plane of a streak camera (Hamamatsu C5680). The mirror could be retracted in order to allow the light to go into another branch for the fluctuation measurement. An interferometric filter (Melles Griot) with Gaussian transmission curve centered at $632.8 \mathrm{~nm}$ and with peak transmission of 55\% selected the photons within a bandwidth of $1 \mathrm{~nm}$ FWHM. The much smaller bandwidth of the filter with respect to the spectral amplitude of the light at BL 7.2 conforms to the other measurement theory requirement. The first lens focal length was chosen for keeping the angle between the incoming photon trajectories and the normal to the filter plane small enough to avoid broadening of the filter bandwidth. Downstream of the filter, the transmitted photons were finally focused by a microscope objective (Edmund DIN 10, F 0.25) on the $0.2 \mathrm{~mm}^{2}$ sensitive area of a silicon avalanche photodiode (APD) (Perkin Elmer C30902S, gain 250, $600 \mathrm{MHz}$ bandwidth, $60 \%$ quantum efficiency at $632.8 \mathrm{~nm}$ ). Such APD has a breakdown voltage of $-225 \mathrm{~V}$ and was typically operated with a bias of $-238 \mathrm{~V}$. The signal from the photodiode was amplified (Hamamatsu C5594, $50 \mathrm{k}-1$. $5 \mathrm{GHz}$ bandwidth, $36 \mathrm{~dB}$ gain) and sent to a digital oscilloscope (LeCroy Wavepro 7300 A, $3 \mathrm{GHz}$ bandwidth and 20 Gsamples/ sec ) for data recording and analysis. The oscilloscope was triggered with the $\sim 1.5 \mathrm{MHz}$ revolution clock of the ALS.

Figure 2 shows the typical signal visible at the scope when measuring the light from a single passage of a single ALS bunch. The rms length of the electron beam is expected to be $\sim 25$ ps so that the shape of the pulse in Fig. 2 is totally defined by the bandwidth of the measurement system. The oscilloscope was set in order to measure the areas $\mathrm{S}_{\mathrm{AB}}$ of the signal between points $\mathrm{A}$ and $\mathrm{B}$, and $\mathrm{S}_{\mathrm{CD}}$ between points $\mathrm{C}$ and $\mathrm{D}$ in Fig. 2. $\mathrm{S}_{\mathrm{AB}}$ is proportional to the number of photons impinging on the detector plus the contribution due to the noise in the signal, while $\mathrm{S}_{\mathrm{CD}}$ is a measure of this noise contribution. The lengths of the segments $\mathrm{AB}$ and $\mathrm{CD}$ were set to be equal.

The statistical error in a variance measurement when $N_{S}$ samples are collected is given by

$$
\sigma_{\delta^{2}} / \delta^{2}=\sqrt{2 /\left(N_{S}-1\right)}
$$

The scope was set to calculate the average values for $\mathrm{S}_{\mathrm{AB}}$ and $S_{C D}$ and their standard deviations over 5000 samples per bunch length measurement in order to keep the statistical error at $\sim 2 \%$ according to Eq. (21). In this configuration, the average energy $\langle\mathcal{E}\rangle$ radiated per passage by the electron beam is proportional to $\left\langle\mathrm{S}_{\mathrm{AB}}\right\rangle-\left\langle\mathrm{S}_{\mathrm{CD}}\right\rangle$, while its variance $\sigma_{\mathcal{E}}^{2}$ is proportional to $\sigma_{\mathrm{S}_{\mathrm{AB}}}^{2}-\sigma_{\mathrm{S}_{\mathrm{CD}}}^{2}$. As an example, the amplitudes of such quantities for the case shown in Fig. 2 were $\left\langle\mathrm{S}_{\mathrm{AB}}\right\rangle \cong 120 \mathrm{pV} \mathrm{s}, \quad \sigma_{\mathrm{S}_{\mathrm{AB}}} \cong 10.2 \mathrm{pV} \mathrm{s}$, $\left\langle\mathrm{S}_{\mathrm{CD}}\right\rangle \cong 0.4 \mathrm{pV} \mathrm{s}$, and $\sigma_{\mathrm{S}_{\mathrm{CD}}} \cong 1.8 \mathrm{pV}$ s for a bunch current of $\cong 3 \mathrm{~mA}$ ( $\cong 2 \mathrm{nC})$.

By comparing different amplitude signals, we also verified that the shape of the signal itself did not depend on the amplitude.

The collection of the 5000 samples required $\sim 1$ minute. This value was dominated by the time required for the digital scope to perform the signal acquisition and the data analysis. While very useful and flexible during this proof of principle experiment, there is no real need for such a complex and expensive instrument in a real operational configuration of the monitor. In fact, commercial gated charge to digital converters can operate at several hundred $\mathrm{kHz}$ repetition rates, and by using such components the time per measurement can be reduced by at least 3 orders of magnitude.

BL 7.2 belongs to the previously described category of beam lines, where the radiation angular distribution can be

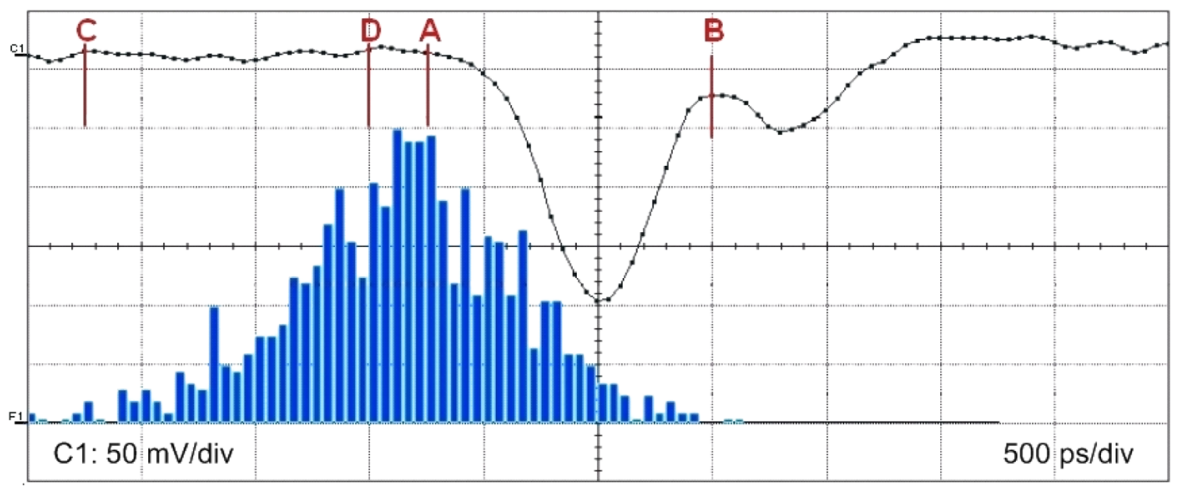

FIG. 2. (Color) Oscilloscope window showing the single passage track of a typical signal from the photodetector and the histogram of $\sim 5000$ measured values of the signal area between points A and B. Another histogram (not shown) of the value of the signal area between points $\mathrm{C}$ and $\mathrm{D}$ was calculated as well. The analysis of such histograms allows the distinction between the fluctuation contribution due to the bunch distribution from that due to other sources of noise (see text). 
well represented by the $[\sin (\xi) / \xi]^{2}$ function. Following the indications at the end of the previous section we obtained, for $\lambda_{0}=632.8 \mathrm{~nm}$ and for the acceptances of BL7.2, $\sigma_{x c} \simeq 29.8 \mu \mathrm{m}$ and $\sigma_{y c} \simeq 59.5 \mu \mathrm{m}$. The measurements were performed at the two beam energies of 1.2 and $1.9 \mathrm{GeV}$. The rms beam sizes at BL 7.2 source point were $\sigma_{x} \simeq 64.8 \mu \mathrm{m}$ and $\sigma_{y} \simeq 6.3 \mu \mathrm{m}$ at $1.2 \mathrm{GeV}$ and $\sigma_{x} \simeq 103.0 \mu \mathrm{m}$ and $\sigma_{y} \simeq 10.0 \mu \mathrm{m}$ at $1.9 \mathrm{GeV}$.

Equations (14) and (17) were derived in a purely classical field theory framework. In the case where the number of photons per pulse $N_{p}$ is not sufficiently large, the proper quantum treatment requires the addition to the right-hand side of Eqs. (14) and (17) of another fluctuation term $1 /\left\langle N_{p}\right\rangle$ due to the shot noise contribution, with $\left\langle N_{p}\right\rangle$ the average number of photons impinging on the detector. Moreover, photodiodes, avalanche photodiodes, and photomultipliers all exploit stochastic phenomena for the photon-electron conversion and amplification. This must be accounted for by using a modified shot noise term $\varsigma^{2} /\left\langle N_{p}\right\rangle=F /\left(Q_{E}\left\langle N_{p}\right\rangle\right)$, where $F$ is the excess noise factor, a quantity $\geq 1$ related to the amplification process in photomultipliers and avalanche photodiodes, that depends on the detector gain and on the operation frequency, and $Q_{E}$ is the detector quantum efficiency at the operation frequency.

Accounting for this contribution and indicating with $\delta_{M}^{2}$ the measured fluctuation variance, Eq. (20) can be modified to finally give the rms length of the bunch:

$\sigma_{t}^{2}=\frac{1}{4 \sigma_{\omega}^{2}}\left[\left(\delta_{M}^{2}-\frac{\varsigma^{2}}{\left\langle N_{p}\right\rangle}\right)^{-2}\left(1+\frac{\sigma_{x}^{2}}{\sigma_{x c}^{2}}\right)^{-1}\left(1+\frac{\sigma_{y}^{2}}{\sigma_{y c}^{2}}\right)^{-1}-1\right]$.

The $s^{2}$ term can be easily measured by performing two or more measurements of $\delta_{M}^{2}$ for the same bunch length but for different numbers of photons impinging on the detector (using neutral density filters, for instance). During the measurements, all the terms on both sides of (22) remain the same with the exception of the shot noise term. From that, and considering that $\left\langle N_{p}\right\rangle \propto\left(\left\langle\mathrm{S}_{\mathrm{AB}}\right\rangle-\left\langle\mathrm{S}_{\mathrm{CD}}\right\rangle\right), \mathrm{s}^{2}$ can be evaluated. The described $s^{2}$ measurement needs to be performed only once during the characterization of the experimental setup.

Figure 3 shows two examples of measurements performed for different beam conditions. The bunch length as a function of the current in the bunch was measured by the fluctuation monitor at 1.2 and $1.9 \mathrm{GeV}$. For each current value, streak camera measurements were also performed right after the fluctuation measurement. The agreement between the two sets of data is remarkably good, especially considering that no parameter has been adjusted to match the data. All measurements show the increase of the beam size as the current per bunch increases due to the collective effects acting on the bunch. The typical rms difference between the streak camera and the fluctuation data was
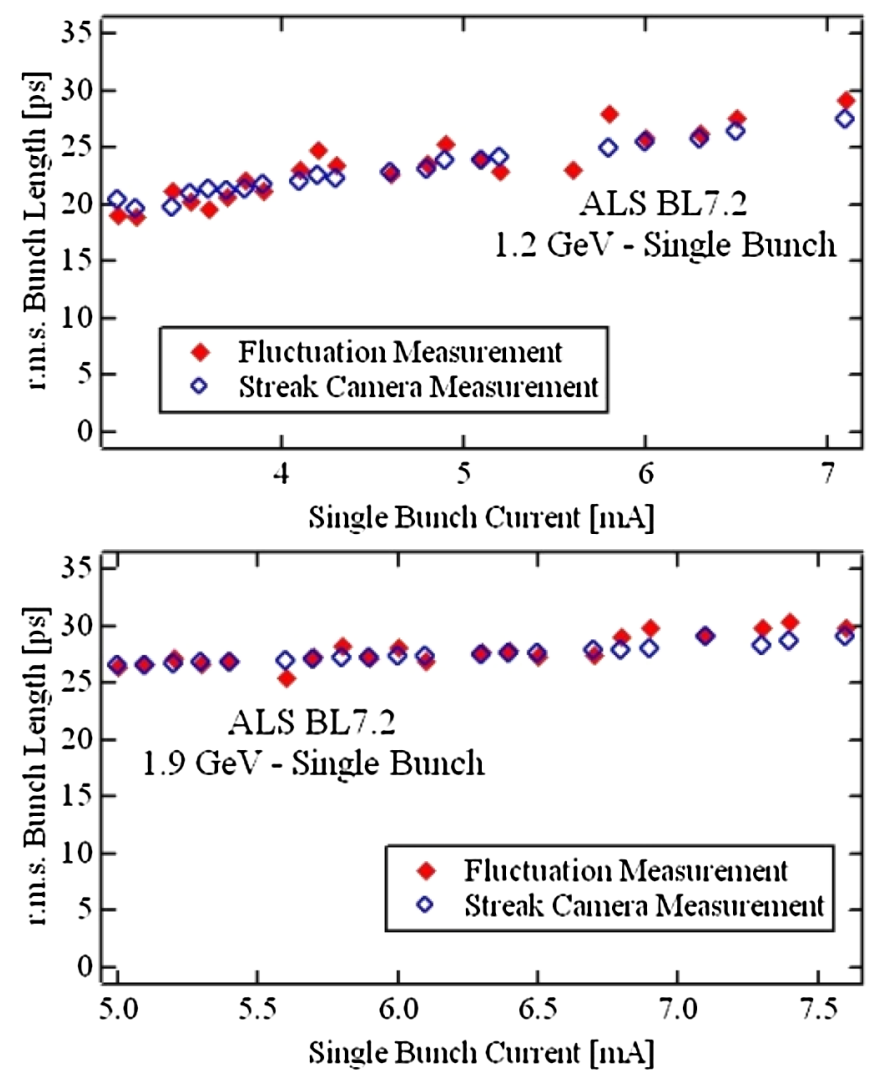

FIG. 3. (Color) Examples of fluctuation and streak camera bunch length measurements at the ALS for different beam parameters.

$\sim 4 \%$. This is larger than the $2 \%$ statistical component calculated by (21). The extra contribution is partially due to the experimental error in the streak camera measurements and also to the fact that the shot noise term during our measurements was comparable to $\delta^{2}$.

In the measurements shown in Fig. 3, the bunch length ranges from $\simeq 20$ to $30 \mathrm{ps} \mathrm{rms}$, and the single bunch current ranges from $\simeq 3$ to $7.5 \mathrm{~mA}$ ( $\simeq 2$ to $5 \mathrm{nC}$ per bunch). The bunch length of the ALS at the normal $1.9 \mathrm{GeV}$ operation energy depends weakly on the bunch current, and the particular ALS lattice does not allow an easy use of the momentum compaction for controlling the bunch length. For that reason, we used the beam energy and, by lowering it down to $1.2 \mathrm{GeV}$, we were able to decrease the bunch length down to $\simeq 20$ ps. Lower energies with stable beam conditions are difficult to obtain at the ALS.

Figure 2 shows the APD signal for the $3 \mathrm{~mA}$ case, the lowest single bunch current measured. The signal to noise ratio was still large and did not limit the capability of measuring lower currents. On the other hand, the shot noise term in Eq. (22) approached the magnitude of the fluctuation term due to the bunch distribution. For lower bunch charges the shot noise contribution becomes stronger and would start affecting the experimental accuracy of the measurement. 
We will now show how this limitation depends on the particular choice of the photodetector used for the measurements. For a good measurement accuracy, we prefer the fluctuation term due to the bunch distribution to be larger than the shot noise term:

$$
\delta^{2} \gtrsim \frac{\varsigma^{2}}{\left\langle N_{p}\right\rangle}=\frac{F\left(G, \omega_{0}\right)}{Q_{E}\left(\omega_{0}\right)} \frac{1}{\left\langle N_{p}\right\rangle},
$$

where $\omega_{0}$ is the frequency of operation and $G$ is the detector gain. The average number of electrons impinging on the detector can be written as

$$
\left\langle N_{p}\right\rangle=a\left(\omega_{0}\right) N \sigma_{\omega} \eta_{\mathrm{BL}},
$$

where $N$ is the number of electrons per bunch, $\eta_{\mathrm{BL}}$ the experimental setup transmission efficiency (filters, beam line viewports, $\ldots)$, and $a\left(\omega_{0}\right)$ is a quantity defined by $a\left(\omega_{0}\right)=\int_{\Omega_{B L}} \frac{\partial N_{p}\left(\Omega, \omega_{0}\right)}{\partial \Omega \partial \omega} d \Omega$, where $\Omega_{B L}$ is the solid angle acceptance of the experimental setup and $\frac{\partial N_{p}\left(\Omega, \omega_{0}\right)}{\partial \Omega \partial \omega}$ is the photon distribution for the radiation from a single electron calculated at $\omega_{0}$. Equation (24) has been derived assuming that $\frac{\partial N_{p}}{\partial \Omega \partial \omega}$ is a slow-varying function of $\omega$ over the interval $\sigma_{\omega}$ around $\omega_{0}$.

Let $T=\left(1+\sigma_{x}^{2} / \sigma_{x c}^{2}\right)^{-1 / 2}\left(1+\sigma_{y}^{2} / \sigma_{y c}^{2}\right)^{-1 / 2}$. As previously shown, $T$ represents the transverse contribution to the fluctuations, and depends on the beam size at the source point, the radiation process, the beam line acceptance, but does not depend on the detector characteristics. The quantity $T$, whose value ranges between 0 and 1 , implicitly depends on $\omega_{0}$ through the quantities $\sigma_{x c}$ and $\sigma_{y c}$, and for whatever radiation process and beam line aperture configuration, smaller $\omega_{0}$ values always lead to smaller $T$ values. Using the $T$ definition in conjunction with $\sigma_{t c}^{2}=1 /\left(2 \sigma_{\omega}\right)$ in Eq. (20), we obtain $\delta^{2}=T\left(1+4 \sigma_{\omega}^{2} \sigma_{t}^{2}\right)^{-1 / 2}$.

By using this last result, jointly to Eq. (24), in Eq. (23), we finally obtain a criterion for the detector choice:

$$
\frac{F\left(G, \omega_{0}\right)}{Q_{E}\left(\omega_{0}\right)} \lesssim \frac{a\left(\omega_{0}\right) N T \sigma_{\omega} \eta_{\mathrm{BL}}}{\sqrt{1+4 \sigma_{\omega}^{2} \sigma_{t}^{2}}} \simeq \frac{a\left(\omega_{0}\right) N T \eta_{\mathrm{BL}}}{2 \sigma_{t}},
$$

where the approximation in the last term holds when $4 \sigma_{\omega}^{2} \sigma_{t}^{2} \gg 1$.

We now give an approximate expression for Eq. (25) where the dependence of $a\left(\omega_{0}\right)$ on $\omega_{0}$ is made explicit. Using the Weizsäcker-Williams method of the virtual photons [12], it can be derived that the number of photons radiated by a single electron within a bandwidth $\sigma_{\omega}$ at the frequency $\omega_{0}$ is $\sim \alpha \sigma_{\omega} / \omega_{0}$, with $\alpha \simeq 1 / 137$ the fine structure constant. Using this last result and the previous definitions, we obtain that $\left\langle N_{p}\right\rangle \sim \alpha N \eta_{\mathrm{BL}} \sigma_{\omega} / \omega_{0}$, which allows one to rewrite criterion (25) as

$$
\frac{F\left(G, \omega_{0}\right)}{Q_{E}\left(\omega_{0}\right)} \lesssim \frac{\alpha N T \sigma_{\omega} \eta_{\mathrm{BL}}}{\omega_{0} \sqrt{1+4 \sigma_{\omega}^{2} \sigma_{t}^{2}}} \simeq \frac{\alpha N T \eta_{\mathrm{BL}}}{2 \omega_{0} \sigma_{t}} .
$$

Equations (25) and (26) show: a weak (typically negli- gible) dependence on the filter bandwidth $\sigma_{\omega}$; that photodetectors with a small excess noise to quantum efficiency ratio are preferred; and also that shorter bunches are less demanding in terms of photodetectors. Equation (26) also shows that the operation frequency $\omega_{0}$ plays a significant role in the detector choice. Indeed, accounting for the explicit $\omega_{0}^{-1}$ dependence, as well as for the abovementioned implicit dependence of $T$ on $\omega_{0}$, smaller frequencies are preferred, with the condition that a detector with a reasonably small $F / Q_{E}$ ratio can be found at those frequencies. Of course in the selection process, other detector parameters, such as gain and speed, need to be considered as well. For example, higher gains are usually preferable, but the excess noise factor $F$ increases with increasing gains, so the proper tradeoff must be found.

The APD selected for the ALS measurements represents a reasonable tradeoff between, gain, excess noise, quantum efficiency, and speed, that properly conforms to the ALS bunch length range and photon number available at BL 7.2 within the selected photon bandwidth. With this detector, at the wavelength and gain of operation, we had $F / Q_{E} \sim$ $6 / 0.6=10$. Using this value and the ALS numbers in criteria (25) and (26), we obtain that below a single bunch current of $\sim 1-2 \mathrm{~mA}$, the shot noise term starts to become dominant, in reasonable agreement with what we observed experimentally.

Different accelerator applications may require a different detector choice (for example, photomultipliers or different photodiodes), depending on the bunch length to be measured, the number of available photons, etc. For example, for shorter bunches (as in FEL and ERL linear accelerator applications) the requirements on the excess noise to quantum efficiency ratio can be relaxed, or if the same detector is used, then smaller bunch charges can be measured. The bandwidth of the filter can be used instead for controlling the requirements on the detector gain. Indeed, a larger $\sigma_{\omega}$ allows for more photons and requires less detector gain with a beneficial reduction on the excess noise factor.

\section{POSSIBLE UPGRADES AND CONCLUSIONS}

By splitting the light from the source in two paths feeding two measurement systems with different bandpass filters, it is in principle possible to discriminate between the transverse and longitudinal components in Eq. (20). In fact, the longitudinal term in the equation depends only on the bandwidth of the filter while the transverse terms depend only on its central wavelength. For example, using filters with the same central wavelength but different bandwidth and comparing the measurement results from the two branches, the dependence on the transverse plane can be removed. Such a feature can be useful when the transverse beam size changes during the accelerator operation, for example. Conversely, if the two branches have filters with different central frequency but the same bandwidth, 
then the dependence from the longitudinal term can be removed.

We also started to test a configuration where the light from the source is coupled to the system by an optical fiber. In those cases where the light is not readily available outside the accelerator vault, the use of the fiber will allow one to transport the light to the measurement setup in a safe and accessible area outside the accelerator vault.

The technique described in this paper can still be used when the charge fluctuates shot to shot, if for each acquisition, the beam charge is measured and the value is used to normalize the fluctuation measurement. The charge measurement can be done within the system itself by simply taking a fraction of the light upstream of the bandpass filter, and by measuring its intensity with a photodetector. The broadband spectrum of the light at this position ensures that the intensity fluctuations due to the bunch distribution are negligible and that the measured intensity represents an accurate relative measurement of the bunch charge. In a particularly effective implementation of such a scheme, a commercial low cost dichroic beam splitter can be used to separate a portion of the spectrum different from the one used by the fluctuation measurement. In this way the bunch charge measurement can be performed without attenuating the light used in the fluctuation branch and thus avoiding an undesired increase of the shot noise contribution.

In summary, we have demonstrated an absolute bunch length measurement technique based on the analysis of the fluctuations in the incoherent part of the radiation emitted by a particle beam. The scheme that can use any kind of radiation process shows a remarkable simplicity and can be applied to both circular and linear accelerators including cases where the very short length of the bunches makes difficult the use of other techniques.

\section{ACKNOWLEDGMENTS}

The authors want to thank J. Byrd, S. De Santis, J. Frisch, and R. Shelton-Mottsmith for their contributions. This work was supported by the Director, Office of Science, of the U.S. Department of Energy under Contracts No. DE-AC03-76SF00098 and No. DE-AC0376 SF00515.

\section{APPENDIX}

Let us now assume that the beam distribution functions are not Gaussian, and also assume arbitrary angular and frequency distributions of the radiation on the detector. At the same time we assume that the function $T$ [see Eq. (16)] is still factorized as

$$
T(\omega, \boldsymbol{\theta})=T_{0} H(\omega) G(\boldsymbol{\theta}),
$$

where $H$ and $G$ are positive real functions normalized by unity,

$$
\int_{-\infty}^{\infty} H(\omega) d \omega=1, \quad \int G(\boldsymbol{\theta}) d \theta_{x} d \theta_{y}=1 .
$$

We will also assume a narrow bandpass filter and replace $k$ and $k^{\prime}$ in Eq. (14) by $k_{0}$ [see explanation in the text after Eq. (16)].

In this case, it is useful to transform the numerator on the right-hand side of Eq. (14) in terms of the original distribution functions in the beam. For example, the frequency dependent factor in Eq. (14) becomes

$$
\begin{aligned}
& \int_{-\infty}^{\infty} H(\omega) H\left(\omega^{\prime}\right)\left|\hat{f}_{t}\left(\omega-\omega^{\prime}\right)\right|^{2} d \omega d \omega^{\prime} \\
& =\int_{-\infty}^{\infty} H(\omega) H\left(\omega^{\prime}\right) f_{t}(t) f_{t}\left(t^{\prime}\right) e^{i\left(\omega-\omega^{\prime}\right) t-i\left(\omega-\omega^{\prime}\right) t^{\prime}} d \omega d \omega^{\prime} d t d t^{\prime} \\
& =\int_{-\infty}^{\infty}\left|\hat{H}\left(t-t^{\prime}\right)\right|^{2} f_{t}(t) f_{t}\left(t^{\prime}\right) d t d t^{\prime},
\end{aligned}
$$

where $\hat{H}(t)$ is the Fourier transform of $H(\omega)$ :

$$
\hat{H}(t)=\int_{-\infty}^{\infty} H(\omega) e^{i \omega t} d \omega .
$$

Similarly, introducing the Fourier transform of the function $G$,

$$
\hat{G}(r)=\int G(\boldsymbol{\theta}) e^{i k_{0} \boldsymbol{\theta} \cdot r} d \theta_{x} d \theta_{y}
$$

and expressing the spacial part in terms of $\hat{G}$ one finds

$$
\begin{aligned}
\delta^{2}= & \int_{-\infty}^{\infty}\left|\hat{H}\left(t-t^{\prime}\right)\right|^{2} f_{t}(t) f_{t}\left(t^{\prime}\right) d t d t^{\prime} \\
& \times \int\left|\hat{G}\left(\boldsymbol{r}-\boldsymbol{r}^{\prime}\right)\right|^{2} F(\boldsymbol{r}) F\left(\boldsymbol{r}^{\prime}\right) d^{2} r d^{2} r^{\prime} .
\end{aligned}
$$

This equation can be further simplified in some limiting cases. For a wide filter, when the characteristic width $\sigma_{\omega}$ of the function $H(\omega)$ satisfies the condition $\sigma_{\omega} \sigma_{t} \gg 1$ one can replace $\left|\hat{H}\left(t-t^{\prime}\right)\right|^{2}$ by a delta function, $\left|\hat{H}\left(t-t^{\prime}\right)\right|^{2}=$ $A \delta\left(t-t^{\prime}\right)$, with $A=2 \pi \int_{-\infty}^{\infty}|H(\omega)|^{2} d \omega$, which gives

$$
\begin{aligned}
& \int_{-\infty}^{\infty}\left|\hat{H}\left(t-t^{\prime}\right)\right|^{2} f_{t}(t) f_{t}\left(t^{\prime}\right) d t d t^{\prime} \\
& \quad \approx 2 \pi \int_{-\infty}^{\infty}|H(\omega)|^{2} d \omega \int_{-\infty}^{\infty} f_{t}(t)^{2} d t .
\end{aligned}
$$

In the opposite limit $\sigma_{\omega} \sigma_{t} \ll 1$, we have

$$
\begin{aligned}
& \int_{-\infty}^{\infty}\left|\hat{H}\left(t-t^{\prime}\right)\right|^{2} f_{t}(t) f_{t}\left(t^{\prime}\right) d t d t^{\prime} \\
& \quad \approx \int_{-\infty}^{\infty}|\hat{H}(0)|^{2} f_{t}(t) f_{t}\left(t^{\prime}\right) d t d t^{\prime} \approx 1,
\end{aligned}
$$

where we used the condition $\hat{H}(0)=1$ which follows from the normalization (A2), and also took into account the normalization of the distribution function $f_{t}$. 
A similar approach can be used to simplify the spatial part in Eq. (A6), if the angular spread of the limiting aperture $\Delta \theta$ is much larger, or much smaller than $\sigma_{\perp} k_{0}$, where $\sigma_{\perp}$ is the characteristic transverse size of the beam.

[1] M. Zolotorev and G. Stupakov, SLAC Report No. PUB 7132, 1996.

[2] M. Zolotorev and G. Stupakov, in Proceedings of the Particle Accelerator Conference, Vancouver, BC, Canada, 1997 (IEEE, New York, 1997), p. 2180.

[3] J. Krzywinski, E. L. Saldin, E. A. Schneidmiller, and M. V. Yurkov, DESY Report No. TESLA-FEL1997-06, 1997, p. 47.

[4] P. Catravas et al., Phys. Rev. Lett. 82, 5261 (1999).

[5] V. Sajaev, in Proceedings of the European Particle Accelerator Conference, Vienna, 2000 (EPS, Geneva, 2000), p. 1806.
[6] F. Sannibale, M. Zolotorev, D. Filipetto, and G. Stupakov, Proceedings of 2007 Particle Accelerator Conference, Albuquerque, New Mexico, USA, 2007, p. 2261.

[7] Kwang-Je Kim, in Characteristic of Synchrotron Radiation, AIP Conf. Proc. No. 184 (1989), p. 622.

[8] The uncertainty principle requires for a Gaussian distribution that $\sigma_{\omega} \sigma_{t}=1 / 2$.

[9] See, for example, Chap. 3 of R. Loudon, The Quantum Theory of Light (Oxford Science Publication, Oxford, UK, 1983), 2nd ed.

[10] T. Tanabe, M. C. Teich, T. C. Marshall, and J. Galayda, Nucl. Instrum. Methods Phys. Res., Sect. A 304, 77 (1991).

[11] SRW code, http://www.esrf.eu/Accelerators/Groups/ InsertionDevices/Software/SRW.

[12] See, for example, Chap. 15, Sec. 4, of J. D. Jackson, Classical Electrodynamics (Wiley, New York, 1999), 3rd ed. 